Bull. Austral. Math. Soc.

VOL. 38 (1988) [177-189]

\title{
A CLASS OF NONCONVEX FUNCTIONS AND MATHEMATICAL PROGRAMMING
}

\author{
T. WEIR AND V. JEYAKUMAR
}

\begin{abstract}
A class of functions, called pre-invex, is defined. These functions are more general than convex functions and when differentiable are invex. Optimality conditions and duality theorems are given for both scalar-valued and vector-valued programs involving pre-invex functions.
\end{abstract}

\section{INTRODUCTION}

Let $X$ and $Y$ be real normed spaces of any dimension and let $K \subseteq Y$ be a closed convex cone. Let $S \subset X$. The function $f: S \rightarrow Y$ is said to be $K$-convexlike (see for example $[10,13,15])$ if for any $x, y \in S$ and $0 \leqslant \lambda \leqslant 1$ there is a $z \in S$ such that

$$
\lambda f(x)+(1-\lambda) f(y)-f(z) \in K .
$$

If $S$ is a convex set and if $f$ is a $K$-convex function, then clearly $f$ is $K$-convexlike. Any real valued function is $\mathbf{R}_{+}$-convexlike.

Elster and Neshe $[\mathbf{1 0}]$ considered convexlike mathematical programs and obtained a saddlepoint optimality condition. Hayashi and Komiya [13] also considered convexlike mathematical programs and established a theorem of the alternative involving convexlike functions and considered Lagrangian duality.

Following [8], a function $f: S \rightarrow Y$ is called $K$-invex, with respect to a function $\eta: S \times S \rightarrow X$, if, for each $x, y \in S$

$$
f(x)-f(y)-f^{\prime}(y) \eta(x, y) \in K
$$

where $f^{\prime}(y)$ denotes the Fréchet derivative of $f$ at $y$. If $Y=\mathbf{R}$ and $K=\mathbf{P}_{+}$, then $f$ is called invex. Invex functions were first considered by Hanson [11] who showed that if, instead of the usual convexity conditions, the objective function and each of the constraints of a nonlinear program are all invex for the same $\eta(x, y)$ then the sufficiency of the Kuhn-Tucker conditions [17] and weak (Wolfe[24]) duality still holds. Moreover, Craven and Glover [0] (also Ben-Israel and Mond [1], Martin [19]) showed that the class

Received 2 November, 1987

Copyright Clearance Centre, Inc. Serial-fee code: 0004-9729/88 \$A2.00+0.00. 
of real valued invex functions is equivalent to the class of functions whose stationary points are global minima.

Following Ben-Israel and Mond [1] and Hanson and Mond [12] consider a function $f: S \rightarrow Y$ having the property that there exists a function $\eta: S \times S \rightarrow X$ such that, for each $x, y \in S$ and $0 \leqslant \lambda \leqslant 1, y+\lambda \eta(x, y) \in S$ and

$$
\lambda f(x)+(1-\lambda) f(y)-f(y+\lambda \eta(x, y)) \in K .
$$

It is to be observed that if $f$ is Fréchet differentiable and satisfies (1.3) then $f$ also satisfies (1.2). This can be seen by rewriting (1.3) as

$$
\lambda(f(x)-f(y))-[f(y+\lambda \eta(x, y))-f(y)] \in K
$$

and then dividing by $\lambda>0$ and taking the limit as $\lambda \rightarrow 0_{+}$gives

$$
f(x)-f(y)-f^{\prime}(y) \eta(x, y) \in K .
$$

In view of this observation functions satisfying (1.3) will be called $K$-pre-invex. It is to be noted that the set $S$ should have the "connectedness" property that $y+\lambda \eta(x, y) \in$ $S$ for $x, y \in S$ and $0 \leqslant \lambda \leqslant 1$. Note also that if $\eta(x, y) \equiv \alpha(x, y)(x-y)$ where $0<\alpha(x, y) \leqslant 1$ then $S$ should be star-shaped $[\mathbf{1 6}]$.

If $Y=\mathbf{R}$ and $K=\mathbf{R}_{+}$and if $f$ satisfies (1.3) then $f$ will be called pre-invex. If $\eta(x, y)=x-y$ then clearly $f$ is convex and $S$ is a convex set; however there are functions which are pre-invex but not convex. For example, consider the function $f: \mathbf{R} \rightarrow \mathbf{R}$ defined by $f(x)=-|x|$. Then $f$ is not convex but is pre-invex with $\eta$ given by

$$
\eta(x, y)=\left\{\begin{array}{l}
x-y \text { if } x \leqslant 0, \quad y \leqslant 0 \\
x-y \text { if } x \geqslant 0, \quad y \geqslant 0 \\
y-x \text { otherwise. }
\end{array}\right.
$$

It is easy to see that a pre-invex function is also $\mathbf{R}_{+}$-convexlike; however pre-invex functions have some interesting properties that are not generally shared by the wider class of convexlike functions. For example, as for convex functions, every local minimum of a pre-invex function is a global minimum and non-negative linear combinations of pre-invex functions are pre-invex.

Thеонем 1.1. Let $f: S \rightarrow \mathbf{R}$ be pre-invex. Then any local minimum of $f$ is a global minimum.

Proof: Let $f$ attain a local minimum $p \in S$; assume that $f(x)<f(p)$ for some $x \in S$. Since $f$ is pre-invex there exists $\eta: S \times S \rightarrow X$ such that

$$
\lambda f(x)+(1-\lambda) f(p) \geqslant f(p+\lambda \eta(x, p)), \quad 0 \leqslant \lambda \leqslant 1 .
$$


Thus

$$
f(p+\lambda \eta(x, p))-f(p) \leqslant \lambda[f(x)-f(p)]<0
$$

for arbitrarily small $\lambda>0$, contradicting the local minimum.

Theorem 1.2. Let $f_{i}: S \rightarrow \mathbf{R}$ be pre-invex (with respect to $\eta$ ), $i=1,2, \ldots, k$. Then $\sum_{i=1}^{k} y_{i} f_{i}(x)$ is pre-invex (with respect to $\eta$ ), where $y_{i} \geqslant 0, i=1,2, \ldots, k$.

ProOF:

$$
\begin{gathered}
\lambda \sum_{i=1}^{k} y_{i} f_{i}(x)+(1-\lambda) \sum_{i=1}^{k} y_{i} f_{i}(y) \\
=\sum_{i=1}^{k} y_{i}\left\{\lambda f_{i}(x)+(1-\lambda) f_{i}(y)\right\} \geqslant \sum_{i=1}^{k} y_{i} f_{i}(y+\lambda \eta(x, y)) .
\end{gathered}
$$

Consider now a function $f: S \rightarrow Y$. Then $f$ is directionally differentiable at $a \in S$ if, for each $x \in S$, the limit

$$
f^{\prime}(a, x)=\lim _{\alpha \neq 0} \alpha^{-1}[f(a+\alpha x)-f(a)]
$$

exists in $Y$. When $Y=\boldsymbol{R}$ this reduces to the usual definition of directional differentiability.

Thoerem 1.3. Let $f: S \rightarrow \dot{Y}$ be directionally differentiable at each point in each direction, and let $f$ be $K$-pre-invex. Then, for all $a, x \in S$,

$$
f(x)-f(a)-f^{\prime}(a, \eta(x, a)) \in K
$$

Proof: Since $f$ is $K$-pre-invex then for all $a, x \in S$ there exists $\eta(x, a)$ such that

$$
f(x)-f(a)-\lambda^{-1}[f(a+\lambda \eta(x, a))-f(a)] \in K .
$$

Letting $\lambda \downarrow 0$ gives the desired result.

\section{Pre-inVeX Functions and Mathematical Programming}

In this section we discuss some applications of pre-invex functions in mathematical programming. The discussion begins with an alternative theorem due to Hayashi and Komiya [13] (see also Jeyakamur [15]) established for convexlike functions which, of course, must also hold for pre-invex functions. From this alternative theorem we will deduce a saddlepoint theorem and Lagrangian duality theorem. We will also discuss Fritz John and Kuhn-Tucker conditions in terms of directional derivatives of the objective and constraint functions. 
TheOREM 2.1. Let $X, Y$ be real normed linear spaces and let $K$ be a closed convex cone in $Y$ with nonempty interior; let $S \subseteq X$. Suppose that $f: S \rightarrow Y$ is $K$-pre-invex. Then exactly one of the following holds:

(i) $(\exists x \in S)-f(x) \in$ int $K$,

(ii) $\left(\exists 0 \neq p \in K^{*}\right)(p f)(S) \subseteq \mathbf{R}_{+}$,

where int denotes interior and $K^{*}$ is the dual cone of $K$.

This result is a special case of the convexlike results of Hayashi and Komiya [13] and Jeyakumar [15]. The following saddlepoint and duality theorems follow from the alternative theorem in a manner analogous to those in [15] for convexlike programs.

Consider the following programs:

(P) minimise $f(x)$ subject to $-g(x) \in K$,

where $X, Y$ are normed linear spaces, $K \subseteq Y$ is a closed convex cone with nonempty interior; $S \subset X, f: S \rightarrow \mathbb{A}$ is pre-invex (with respect to $\eta$ ) and $g: S \rightarrow Y$ is $K$ pre-invex (with respect to $\eta$ ). The hypotheses stated here will be assumed to hold throughout the remainder of this section.

(D) maximise $\varphi(v)$ subject to $v \in K^{\star}$, where $\varphi(v)=\inf _{x \in S}\{f(x)+v g(x)\}$.

The program (P) is said to satisfy the generalised Slater condition if there is $\bar{x} \in S$ such that $-g(\bar{x}) \in \operatorname{int} K$.

TheOREM 2.2. If $(P)$ attains a minimum at $x=x_{0} \in S$ and if the generalised Slater condition is satisfied, then there is a $v_{0} \in K^{\star}$ such that the Lagrangian $\psi(x, v)=$ $f(x)+v g(x)$ satisfies the saddlepoint condition at $\left(x_{0}, v_{0}\right)$ :

$$
\left(\forall x \in S, \quad \forall v \in K^{\star}\right), \quad \psi\left(x_{0}, v\right) \leqslant \psi\left(x_{0}, v_{0}\right) \leqslant \psi\left(x, v_{0}\right)
$$

Furthermore, if $(2.1)$ is satisfied for some $\left(x_{0}, v_{0}\right)$ then $x_{0}$ is a minimum for $(P)$.

Remark. The saddlepoint condition (2.1) is sufficient without any pre-invexity assumptions.

Theorem 2.3. Assume $f$ is pre-invex (with respect to $\eta$ ) and that $g$ is $K$ pre-invex (with respect to $\eta$ ). Assume also that $(P)$ satisfies the generalised Slater condition. Then $(D)$ is a dual for $(P)$.

We now turn our attention to local necessary optimality conditions and in particular the Fritz John and Kuhn-Tucker conditions. We consider the program (P) where now $S \subseteq X$ is an open set and where $f$ and $g$ are directionally differentiable at each point in each direction. 
Theorem 2.4. For the program $(P)$ let $f$ and $g$ be directionally differentiable. Assume, also, that $f$ and $g$ are pre-invex and $K$-pre-invex (with respect to $\eta$ ) respectively and that $(P)$ attains a minimum at $x=x_{0}$. Then there exist $\tau \in \mathbf{P}_{+}$and $\lambda \in K^{\star}$ not both zero such that

$$
\begin{gathered}
(\tau f+\lambda g)^{\prime}\left(x_{0}, x\right) \geqslant 0 \quad \forall x \in S, \\
\lambda g\left(x_{0}\right)=0 .
\end{gathered}
$$

Proof: Since $-g(x) \in K$ implies that $f\left(x_{0}\right)-f(x) \leqslant 0$. for all $x \in S$, then there is no solution $x \in S$ to the system

$$
-\left(f(x)-f\left(x_{0}\right), g(x)\right) \in \operatorname{int}\left(\mathbb{R}^{+} \times K\right) .
$$

Then by Theorem 2.1 there exists $\tau \in \mathrm{R}_{+}, \lambda \in K^{\star}$, not both zero, such that for all $x \in S$

$$
\tau f(x)+\lambda g(x) \geqslant \tau f\left(x_{0}\right)
$$

Since $-g\left(x_{0}\right) \in K, \lambda g\left(x_{0}\right)=0$. Therefore, for all $x \in S$,

$$
\tau f(x)+\lambda g(x)-\left[\tau f\left(x_{0}\right)+\lambda g\left(x_{0}\right)\right] \geqslant 0 .
$$

This gives, for all $x \in S$,

$$
(\tau f+\lambda g)^{\prime}\left(x_{0}, x\right) \geqslant 0
$$

since the functions are directionally differentiable.

The Fritz John conditions (2.2) and (2.3) lead to appropriate Kuhn-Tucker conditions under any assumption that implies $r \neq 0$. Moreover, the Kuhn-Tucker conditions are also sufficient.

Theorem 2.5. For the program $(P)$, let $f$ and $g$ be directionally differentiable at each point in each direction. Assume also that $f$ is pre-invex (with respect to $\eta$ ) and that $g$ is $K$-pre-invex (with respect to $\eta$ ) and that the generalised Slater condition is satisfied. Then $(P)$ attains a minimum at $x=x_{0}$ if and only if there exists $\lambda \in K^{*}$ such that

$$
\begin{aligned}
(f+\lambda g)^{\prime}\left(x_{0}, x\right) & \geqslant 0 \quad \forall x \in S \\
\lambda g\left(x_{0}\right) & =0 .
\end{aligned}
$$

Proof: ( $\Longrightarrow$ ) Assume that $(\mathrm{P})$ attains a minimum at $x=x_{0}$. Then the Fritz John conditions (2.2) and (2.3) must be satisfied at $x=x_{0}$ for some $\tau \in \mathbf{R}_{+}$, $\lambda \in K^{\star}$ not both zero. If $\tau=0$, then $\lambda \neq 0$ and $(\lambda g)^{\prime}\left(x_{0}, x\right) \geqslant 0$ for all $x \in S$ 
and $\lambda g\left(x_{0}\right)=0$. Since $g$ is $K$-pre-invex it follows that $\lambda g(x) \geqslant \lambda g\left(x_{0}\right)=0$; this contradicts the generalised Slater condition by Theorem 2.1. Hence $\tau \neq 0$ and we may assume $\tau=1 ;(2.4)$ and (2.5) then follow directly from (2.2) and (2.3).

$(\Longleftarrow)$ Let $x$ be feasible and assume that (2.4) and (2.5) are satisfied. Then

$$
\begin{aligned}
f(x)-f\left(x_{0}\right) & \geqslant f^{\prime}\left(x_{0}, \eta\left(x, x_{0}\right)\right) \quad(\text { by Theorem 1.3) } \\
& \geqslant-(\lambda g)^{\prime}\left(x_{0}, \eta\left(x, x_{0}\right)\right) \quad(\text { by }(2.4)) \\
& \geqslant-\lambda\left(g(x)-g\left(x_{0}\right)\right) \quad(\text { since } g \text { is } K \text {-pre-invex) } \\
& =-\lambda g(x) \quad\left(\text { since } \lambda g\left(x_{0}\right)=0\right) \\
& \geqslant 0 \quad\left(\text { since } \lambda \in K^{\star},-g(x) \in K\right) .
\end{aligned}
$$

Hence $f(x) \geqslant f\left(x_{0}\right)$.

It is to be noted that, for a related convexlike program, the Kuhn-Tucker conditions may not be sufficient for a minimum. However, for pre-invex programs the Kuhn-Tucker conditions are both necessary and sufficient. This extends a well-known result in convex programming (see for example Rockafellar [20]).

Now, in relation to $(\mathrm{P})$ consider the program

(D1) maximise $f(u)+\lambda g(u)$, subject to $(f+\lambda g)^{\prime}(u, x) \geqslant 0, \quad \lambda \in K^{*}, u \in S . \forall x \in S$.

We show that (D1) is a dual to $(P)$.

Theorem 2.6. In $(P)$, let $f$ and $g$ be directionally differentiable at each point in each direction. Let $f$ be pre-invex (with respect to $\eta$ ) and let $g$ be $K$-pre-invex (with respect to $\eta$ ). Let $(P)$ attain a minimum at $x_{0} \in S$, and let the Kuhn-Tucker conditions (2.4) and (2.5) hold at $x_{0}$. Then (D1) is a dual to $(P)$.

Proof: Let $-g(x) \in K$ and let $\lambda \in K^{\star}$. Then

$$
\begin{aligned}
f(x)- & {[f(u)+\lambda g(u)] \geqslant f^{\prime}(u, \eta(x, u))-\lambda g(u) \text { (by Theorem 1.3) } } \\
& \geqslant-\lambda\left(g(u)+g^{\prime}(u, \eta(x, u))\right) \text { (substituting from the constraint of (D1)) } \\
& \geqslant-\lambda g(x)(\text { since } \lambda g(\cdot) \text { is pre-invex and by Theorem 1.3) } \\
& \geqslant 0 \text { since }-g(x) \in K \text { and } \lambda \in K^{\star} .
\end{aligned}
$$

This proves weak duality. Now, from the Kuhn-Tucker conditions for (P), there is a $\bar{\lambda} \in K^{*}$ with

$$
(f+\bar{\lambda} g)^{\prime}\left(x_{0}, x\right) \geqslant 0 \text { and } \bar{\lambda} g\left(x_{0}\right)=0
$$

so $\left(x_{0}, \bar{\lambda}\right)$ satisfies the constraints of (D1) and

$$
\max (\mathrm{D} 1) \geqslant f\left(x_{0}\right)+\bar{\lambda} g\left(x_{0}\right)=f\left(x_{0}\right)=\min (\mathrm{P}) .
$$

This, with weak duality, shows $\left(x_{0}, \bar{\lambda}\right)$ is optimal for (D1). 


\section{Pre-invex functions and Vector-VAlued programming}

Let $X$ and $Y$ be real normed spaces of any dimension and let $S \subseteq X$. Let $f: S \rightarrow Y$ and let $Q \subset Y$ be a closed convex cone. Consider the vector valued problem

$$
\text { minimise } f(x) \text { subject to } x \in T
$$

where $T \subset S$. The problem (3.1) has a weak minimum at $x=x_{0} \in T$ (see for example $[3,5,6])$ if there exists no $x \in T$ for which

$$
f\left(x_{0}\right)-f(x) \in \operatorname{int} Q,
$$

where int denotes interior. Local weak minima may be obtained from the above with $T \cap N$ replacing $T$ where $N$ is a sufficiently small neighbourhood of $x_{0}$.

Consider the problem

$$
\text { (P1) minimise } f(x) \text { subject to }-g(x) \in K
$$

where $X, Y, Z$ are real normed vector spaces with $S \subseteq X ; Q \subseteq Y$ and $K \subseteq Z$ are closed convex cones, and $f: S \rightarrow Y, g: S \rightarrow Z$. The hypotheses stated will be assumed to hold throughout this section.

For vector-valued problems it is natural to study a vector-valued Lagrangian generalising the usual scalar Lagrangian. For convex problems this has been done in finite dimensions for Pareto optima by Tanino and Sarawagi [21] and White [23] and for weak optima in infinite dimensions by Weir, Mond and Craven [22]. Other approaches, using matrix Lagrange multipliers, have been given by Bitran [2], Ivanov and Nehse [14] for finite dimensions and by Corely [4] for infinite dimensions.

In this section we will use the same vector-valued Lagrangian as in [22] and regard $f$ and $g$ as $Q$-pre-invex and $K$-pre-invex functions respectively. We will establish necessary and sufficient conditions for weak minimisation and duality theorems.

First we need some preliminaries. Let $X, Y, Z$ be real normed spaces and $S$ a subset of $X$. Let $P \subseteq Z$ be a convex cone and let $W$ be a set in $Z$. A point $w_{0} \in W$ is called an extreme point (see for example [21]) of $W$ with respect to $P$ if there is no $w \in W, w \neq w_{0}$, such that $w-w_{0} \in \operatorname{int} P$. The problem (3.1) may thus be interpreted as that of finding all the extreme points of $-f(T)$ with respect to $Q$.

For the problem (P1) with int $Q \neq \phi$ define a Lagrangian $L_{r}: X \times K^{\star} \rightarrow Y$ by $L_{r}(x, v)=f(x)+v g(x) r$, for a fixed $r \in \operatorname{int} Q$. The point $\left(x_{0}, v_{0}\right)$ will be called a saddlepoint of $L_{r}(x, v)$ if for all $x \in S, v \in K^{\star}$,

$$
\begin{aligned}
& L_{r}\left(x_{0}, v\right)-L_{r}\left(x_{0}, v_{0}\right) \notin \operatorname{int} Q \\
& L_{r}\left(x_{0}, v_{0}\right)-L_{r}\left(x, v_{0}\right) \notin \operatorname{int} Q
\end{aligned}
$$


We will now give sufficient and necessary optimality conditions for (P1) in terms of a vector-valued Lagrangian. As in the case of scalar programming, if $\left(x_{0}, v_{0}\right)$ is a solution of (3.2) and (3.3) for some $r \in$ int $Q$ then $x_{0}$ is an optimal solution for (P1). This is established in [22, Theorem 2]. However, as in[22], if $x_{0}$ is an optimal solution of (P1) a constraint qualification and convexity is required to assure the existence of $v_{0}$ such that $\left(x_{0}, v_{0}\right)$ is a solution of (3.2) and (3.3). Here we will show that this convexity requirement can be weakened to pre-invexity.

TheOREM 3.2. Let $f$ be $Q$-pre-invex and $g K$-pre-invex. Suppose $x_{0}$ is an optimum solution for (P1) such that $\tau f\left(x_{0}\right) \leqslant \tau f(x)$ for some $0 \neq \tau \in Q^{\star}$ and all feasible $x \in S$. If the generalised Slater condition is satisfied then there exists $v_{0} \in I^{-\star}$ such that the saddlepoint conditions (3.2) and (3.3) hold for some $r \in \operatorname{int} Q$ and $v_{0} g\left(x_{0}\right)=0$.

Remark. A sufficient condition guaranteeing the existence of $0 \neq \tau \in Q^{\star}$ such that $\tau f\left(x_{0}\right) \leqslant \tau f(x)$ for all feasible $x \in S$ is that $f$ is $Q$-pre-invex, $g$ is $K$-pre-invex, (P1) attains a weak local minimum at $x=x_{0}$ and that for some sufficiently small neighbourhood $N$ of $x_{0}$ the set

$$
C=\left\{\beta\left(f(x)-f\left(x_{0}\right)\right): \beta \in \mathbf{R}_{+}, \quad x \in F \cap N\right\}
$$

is convex, where $F=\{x:-g(x) \in K\}[7]$.

Proof: From the assumptions $x_{0}$ is a solution of the scalar mininisation problem

$$
\text { minimise } \tau f(x) \text { subject to }-g(x) \in K
$$

and, since $\tau f$ is pre-invex and $g$ is $K$-pre-invex, Theorem 2.2 gives $\tau\left(f\left(x_{0}\right)+v_{0} g\left(x_{0}\right) r\right)$ $\leqslant \tau\left(f\left(x_{0}\right)+v_{0} g\left(x_{0}\right) r\right) \leqslant \tau\left(f(x)+v_{0} g(x) r\right)$ for some $r \in \operatorname{int} Q$ chosen such that $\tau r=$ 1. If (3.2) and (3.3) did not hold then

$$
\begin{gathered}
\tau\left(f\left(x_{0}\right)+v g\left(x_{0}\right) r-\left(f\left(x_{0}\right)+v_{0} g\left(x_{0}\right) r\right)\right)>0 \text { and } \\
\tau\left(f\left(x_{0}\right)+v_{0} g\left(x_{0}\right) r-\left(f(x)+v_{0} g(x) r\right)\right)>0
\end{gathered}
$$

a contradiction.

Consider the two problems

(A) minimise $\Psi(x)$ (weakly with respect to some cone $C$ ) subject to $x \in F$ and

(B) maximise $\Phi(y)$ (weakly with respect $C$ ) subject to $y \in G$. 
Problem (B) will be called a dual of (A) if ([6]) there holds.

(i) (weak duality) $\Psi(x)-\Phi(y) \notin-$ int $C$ whenever $x \in F$ and $y \in G$; and

(ii) (strong duality) if (A) attains a weak ninimum at some point $x=a$, then (B) attains a weak maximum at some point $y=b \in G$ and $\Psi(a)=\Phi(b)$.

In relation to $(\mathrm{P} 1)$ consider the problem

$\left(D^{\prime}\right)$ maximise $\Xi=\left\{\xi \in Y:\left(\exists 0 \neq \tau \in Q^{\star}, \quad v \in S^{\star}\right), \quad \tau \xi=\inf \left\{\tau f(z): z \in S_{0}\right\}\right\}$.

The maximisation problem $\left(D^{\prime}\right)$ is the problem of finding the extreme points of $\Xi$ with respect to the cone $Q$.

Theorem 3.3. (Weak Duality) Let $x$ be feasible for (P1) and let $\eta \in \Xi$. Then $f(x)-\eta \notin-\operatorname{int} Q$

Proof: For some $0 \neq \tau \in Q^{\star}, v \in S^{\star}, \tau \eta=\inf \left\{\tau f(z)+v g(z): z \in X_{0}\right\}$. Hence $\tau f(x) \geqslant \tau f(x)+v g(x) \geqslant \inf \left\{\tau f(z)+v g(z): z \in S_{0}\right\}=\tau \eta$; so $\tau(f(x)-\eta) \geqslant 0$; thus $f(x)-\eta \notin \operatorname{int} Q$.

Theorem 3.4. (Strong Duality). Let $f$ be $Q$-pre-invex and $g K$-pre-invex. Let $x_{0}$ be a solution to (P1) such that $\tau f\left(x_{0}\right) \leqslant \tau f(x)$ for some $0 \neq \tau \in Q^{\star}$ and all $x \in S$. If the generalised Slater condition is satisfied then there is $\xi_{0} \in \Xi$ such that $f\left(x_{0}\right)=\xi_{0}$ and $\xi_{0}$ is an extreme point of $\Xi$.

Proof: From the assumptions $x_{0}$ is a solution of the scalar minimisation problem:

$$
\text { minimise } \tau f(x) \text { subject to }-g(x) \in K \text {. }
$$

From Theorem 2.3 there exists $v_{0} \in K^{\star}$ such that $v_{0} g\left(x_{0}\right)=0$ and for all $x \in S$

$$
\tau f\left(x_{0}\right)+v_{0} g\left(x_{0}\right) \leqslant \tau f(x)+v_{0} g(x) .
$$

Thus,

$$
\tau f\left(x_{0}\right) \leqslant \inf \left\{\tau f(x)+v_{0} g(x)\right\}=\tau \xi
$$

for some $\xi \in Y$. From weak duality it follows that $\tau f\left(x_{0}\right)=\tau \xi$. If there was no $\xi_{0} \in \Xi$ being an extreme point of $\Xi$ such that $f\left(x_{0}\right)=\xi_{0}$ then there would be $\hat{\xi}_{0} \in \Xi$ such that $\hat{\xi}-f\left(x_{0}\right) \in \operatorname{int} Q$; hence for all $0 \neq \tau \in Q^{*}, \tau \hat{\xi}>\tau f\left(x_{0}\right)$. Thus, since $\hat{\xi} \in \Xi$, for some $\hat{\tau} \in Q^{\star}, \hat{v} \in K^{\star}, \inf \left\{\hat{\tau} f(x)+\hat{v} g(x): x \in S_{0}\right\}=\hat{\tau} \hat{\xi}>\hat{\tau} f\left(x_{0}\right) \geqslant \hat{\tau} f\left(x_{0}\right)+\hat{v} g\left(x_{0}\right)$ which is a contradiction.

We now turn our attention to the problem (P1) where $f$ and $g$ are directionally differentiable on the open set $S$ and discuss necessary and sufficient optimality conditions. 
TheOREM 3.5. For the program (P1), let $f$ and $g$ be directionally differentiable at each point in each direction. Assume that $f$ and $g$ are $Q$-pre-invex and $K$-preinvex respectively, and that $(P 1)$ attains a weak minimum at $x=x_{0}$. Then there exist $\tau \in Q^{\star}$ and $\lambda \in K^{\star}$, not both zero, such that

$$
\begin{gathered}
(\tau f+\lambda g)^{\prime}\left(x_{0}, x\right) \geqslant 0 \quad \forall x \in S, \\
\lambda g\left(x_{0}\right)=0 .
\end{gathered}
$$

Proof: Since $-g(x) \in K$ implies that $f\left(x_{0}\right)-f(x) \notin$ int $Q$ for all $x \in S$, then there is no solution $x \in S$ to the system

$$
-\left(f(x)-f\left(x_{0}\right), g(x)\right) \in \operatorname{int}(Q \times K) .
$$

Then by Theorem 2.1 there exists $\tau \in Q^{\star}$ and $\lambda \in K^{\star}$, not both zero, such that for all $x \in S$

$$
\tau f(x)+\lambda g(x) \geqslant \tau f\left(x_{0}\right) .
$$

Since $-g\left(x_{0}\right) \in K, \lambda g\left(x_{0}\right)=0$. Therefore, for all $x \in S$,

$$
\tau f(x)+\lambda g(x)-\left[\tau f\left(x_{0}\right)+\lambda g\left(x_{0}\right)\right] \geqslant 0 .
$$

This gives that, for all $x \in S$,

$$
(\tau f+\lambda g)^{\prime}\left(x_{0}, x\right) \geqslant 0
$$

since the functions are directionally differentiable.

The Fritz John conditions (3.4) and (3.5) will lead to appropriate Kuhn-Tucker necessary conditions under any assumption giving $\tau \neq 0$. Moreover, the Kuhn-Tucker conditions are also sufficient.

Theorem 3.6. For the program (P1), let $f$ and $g$ be directionally differentiable at each point in each direction. Assume also that $f$ is $Q$-pre-invex and $g K$-preinvex and that the generalised Slater condition is satisfied. Then (P1) attains a weak minimum at $x=x_{0}$ if and only if there exists $0 \neq \tau \in Q^{\star} \lambda \in K^{\star}$ such that:

$$
\begin{gathered}
(\tau f+\lambda g)^{\prime}\left(x_{0}, x\right) \geqslant 0, \quad \forall x \in S, \\
\lambda g\left(x_{0}\right)=0 .
\end{gathered}
$$

Proof: $(\Longrightarrow)$. Assume that $(\mathrm{P})$ attains a weak minimum at $x=x_{0}$. Then the Fritz John conditions (3.4) and (3.5) must be satisfied at $x=x_{0}$, for some $\tau \in Q^{\star}$, $\lambda \in K^{\star}$ not both zero. If $\tau=0$, then $\lambda \neq 0$ and $(\lambda g)^{\prime}\left(x_{0}, x\right) \geqslant 0$ for all $x \in S$, and 
$\lambda g\left(x_{0}\right)=0$. Since $g$ is $K$-pre-invex it follows that $\lambda g(x) \geqslant \lambda g\left(x_{0}\right)=0$ for all $x \in S$; this contradicts the generalised Slater condition by Theorem 2.1. Hence, $\tau \neq 0$, and (3.6) and (3.7) follows.

$(\Longleftarrow)$. Let $x$ be feasible and assume that (3.6) and (3.7) are satisfied. Since $0 \neq \tau \in Q^{\star}$ and $f$ is $Q$-pre-invex, then $\tau f$ is pre-invex. Then

$$
\begin{aligned}
\tau f(x)-\tau f\left(x_{0}\right) & \geqslant(\tau f)^{\prime}\left(x_{0}, \eta\left(x, x_{0}\right)\right) \text { (by Theorem 1.3) } \\
& \geqslant-(\lambda g)^{\prime}\left(x_{0}, \eta\left(x, x_{0}\right)\right) \text { (by (3.6)) } \\
& \geqslant \lambda\left(g(x)-g\left(x_{0}\right)\right)(\text { since } g \text { is } K \text {-pre-invex) } \\
& =-\lambda g(x)\left(\text { since } \lambda g\left(x_{0}\right)=0\right) \\
& \geqslant 0\left(\text { since } \lambda \in K^{\star},-g(x) \in K\right)
\end{aligned}
$$

Hence $f(x)-f\left(x_{0}\right) \notin-\operatorname{int} Q$.

Using the Kuhn-Tucker conditions for (P1) we will be able to establish a duality theorem for $(\mathrm{P} 1)$ and the problem

$$
\begin{aligned}
&\left(\mathrm{D} 1^{\prime}\right) \text { maximise } f(u)+\lambda g(u) r, \\
& \text { subject to }(\tau f+\lambda g)^{\prime}(u, x) \geqslant 0 \quad \forall x \in S, \\
& \quad \tau \in Q^{*}, \lambda \in K^{*}, u \in S, \tau r=1,
\end{aligned}
$$

and $r$ is any fixed element of int $Q$.

THEOREM 3.7. In (P1) let $f$ and $g$ be directionally differentiable at each point in each direction. Let $f$ be $Q$-pre-invex (with respect to $\eta$ ) and let $g$ be $K$-pre-invex (with respect to $\eta$ ). Let $(P 1)$ at tain a weak minimum at $x_{0} \in S$ and let Kuhn-Tucker conditions (3.6) and (3.7) hold at $x_{0}$. Then (D1') is a dual to (P1).

Proof: Let $-g(x) \in K$ and let $\tau \in Q^{\star}, \lambda \in K^{\star}$ and $\tau r=1$. Then

$$
\begin{aligned}
\tau f(x) & -\tau[f(u)+\lambda g(u) r]=\tau f(x)-\tau f(u)-\lambda g(u) \\
& \geqslant(\tau f)^{\prime}(u, \eta(x, u))-\lambda g(u)(\text { by Theorem 1.3) } \\
& \geqslant-(\lambda g)^{\prime}(u, \eta(x, u))-\lambda g(u) \\
& \text { (substituting from the constraints of } \left.\left(\mathrm{D} 1^{\prime}\right)\right) \\
& \geqslant-\lambda g(x) \text { (since } \lambda g \text { is pre-invex and by Theorem 1.3) } \\
& \geqslant 0\left(\text { since }-g(x) \in K \text { and } \lambda \in K^{\star}\right) .
\end{aligned}
$$

Hence $f(x)-[f(u)+\lambda g(u) r] \notin-\operatorname{int} Q$. This proves weak duality. Now, from Kuhn-Tucker conditions for (P1), there is $0 \neq \bar{\tau} \in Q^{\star}, \bar{\lambda} \in K^{\star \star}$ such that $\bar{\tau} r=1$ and $(\bar{\tau} f+\bar{\lambda} g)^{\prime}\left(x, x_{0}\right) \geqslant 0$ and $\bar{\lambda} g\left(x_{0}\right)=0$; so $\left(x_{0}, \bar{\tau}, \bar{\lambda}\right)$ satisfies the constraints of (D1') and the values of $(\mathrm{P} 1)$ and $\left(\mathrm{D} 1^{\prime}\right)$ are equal. This establishes strong duality. 


\section{References}

[1] A. Ben-Isreal and B. Mond, 'What is invexity?', J. Austral. Math. Soc. (Ser. B) 28 (1986), 1-9.

[2] G.R. Bitran, 'Duality in nonlinear multiple criteria optimisation problems', J. Optim. Theory Appl. 35 (1982), 367-406.

[3] J.M. Borweill, Optimisation with Respect to Partial Orderings, D. Phil. Thesis, University of Oxford, 1974 .

[4] B.D. Corely, 'Duality theory for maximizations with respect to cones', J. Math. Anal. Appl. 84 (1982), 560-568.

[5] B.D. Craven, 'Nonlinear programming in locally convex spaces', J. Optim. Theory Appl. 10 (1972), $197-210$.

[6] B.D. Craven, 'Lagrangian conditions and quasiduality', Bull. Austral. Math. Soc. 16 (1977), 325-339.

[7] B.D. Craven, 'Lagrangian conditions, vector-minimization and local duality', Dept. Math. University of Melbourne Research Report 37 (1980).

[8] B.D. Craven, 'Invex functions and constrained local minima', Bull. Aust. Math. Soc. 24 (1981), 357-366.

[8] B.D. Craven and B.M. Glover, 'Invex functions and duality', J. Austral. Math. Soc. (Ser. A) 39 (1985), 1-20.

[10] .H. Elster and R. Nelise, 'Optimality conditions for some nonconvex problems', in Optimization Techniques: Lecture Notes in Control and Information Sciences 23, pp. 1-9 (Springer-Verlag, New York).

[11] M.A. Hanson, 'On sufficiency of the Kuhn-Tucker conditions', J. Math. Anal. Appl. 80 (1982), 545-550.

[12] M.A. Hanson and B. Mond, Convex Transformable Programming Problems and Invexity (Florida State University Statistics Report MT15, 1985).

[13] M. Hayashi and H. Koniya, 'Perfect duality for convexlike programs', J. Optim. Theory Appl. 38 (1980), 179-189.

[14] E.H. Ivanov and R. Nehse, 'Some results on dual vector optimization problems', Optimization 16 (1985), 505-517.

[15] V. Jeyakumar, 'Convexlike alternative theorems and mathematical programming', Optimization 16 (1985), 643-652.

[16] R.N. Kaul and S. Kaur, 'Sufficient optimality conditions using generalized convex functions', Opsearch 19 (1982), 212-224.

[17] H.W. Kuhn and A.W. Tucker, 'Nonlinear programming', in Proceedings of the Second Berkely Symposium on Mathematical Statistics and Probability, J. Neyman (ed), pp. 481-492 (University of California Press, Berkeley, California, 1951).

[18] O.L. Mangasarian, Nonlinear Programming (McGraw-Hill, New York, 1969).

[19] D.H. Martin, 'The essence of invexity', J. Optim. Theory Appl. 47 (1985), 65-76.

[20] R.T. Rockafellar, 'Convex Analysis' (Princeton University Press, Princeton, N.J.).

[21] T. Tannino and Y. Sarawagi, 'Duality theory in multiobjective programming', J. Optim. Theory Appl. 27 (1979), 509-529.

[22] T. Weir, B. Mond and B.D. Craven, 'Weak minimization and duality', Num. Func. Anal. Optim. $\theta$ (1987), 181-192.

[23] D.S. White, 'Vector maximization and Lagrange multipliers', Math. Programming 31 (1985), 192-205.

[24] P. Wolfe, 'A duality theorem for noulinear programming', Quart. Appl. Math. 19 (1961), 239-244. 
Dr V. Jeyakumar

Department of Combinatorics and Optimization Faculty of Mathematics

University of Waterloo

Ontario

Canada N2L 3G1
Dr T. Weir

Department of Mathematics

University College

Australian Defence Force Academy

Campbell, A.C.T. 2600

Australia 